\title{
Comparing the strategies used to maintain the coach-athlete relationship in Japan and the United Kingdom
}

\author{
Daniel Rhind ${ }^{\text {a, }}$, Frank Owusu-Sekyere ${ }^{\text {b }}$, Daichi Ando ${ }^{c}$ \\ a School of Sport, Exercise and Health Sciences, Loughborough University, Leicestershire, UK. \\ b Department of Applied and Human Sciences, Kingston University, Kingston University, Kingston Hill, Kingston upon Thames, UK \\ c Department of Sport and Exercise Psychology, Brunel University, Kingston Ln, London, UK \\ *Corresponding author Email: yd97674@uga.edu \\ DOI: https://doi.org/10.34256/ijpefs2142
}

Received: 01-07-2021, Revised: 04-10-2021; Accepted: 06-10-2021; Published: 09-10-2021

Abstract: The present study explored the strategies used to maintain the quality of the coach-athlete relationship amongst rowers in Japan and the United Kingdom. A total of 93 athletes from Japan $(\mathrm{N}=49)$ and UK $(\mathrm{N}=44)$ completed the Coach Athlete Relationship Maintenance Questionnaire (CARM-Q) and the Athlete Satisfaction Questionnaire (ASQ). The results of T-tests showed that (a) university rowers in the UK were significantly more satisfied with the coach-athlete relationship than those in Japan; (b) the athletes in Japan expressed higher scores on Preventative strategies than the ones in the UK; (c) the athletes in the UK expressed higher scores on all other CARM-Q subscales with the exception of Social Networks. The results of correlation analyses revealed positive associations between the use of maintenance strategies and athlete satisfaction. These findings evidence the importance of coaches using strategies to maintain the effectiveness of their relationship with athletes as well as the importance of researchers taking cultural factors into account.

Keywords: Relationship Maintenance, Satisfaction, Rowing, Culture, Conflict Management

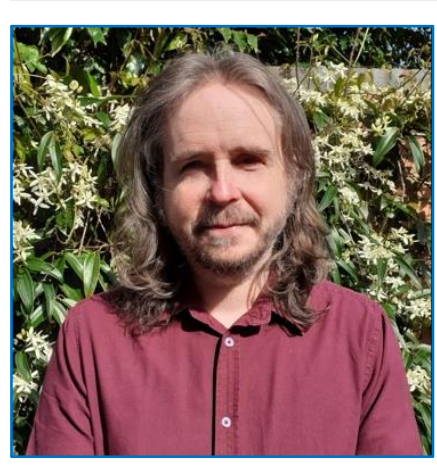

a BSc in social Psychology (Loughborough University). Daniel's research focuses on the realisation of human rights in, around and through sport. His research was fundamental in the development of the International Safeguards for Children in Sport. He also managed Project CARE which involved a global census of elite athletes' experiences regarding how their rights were realised or violated as children in sport.

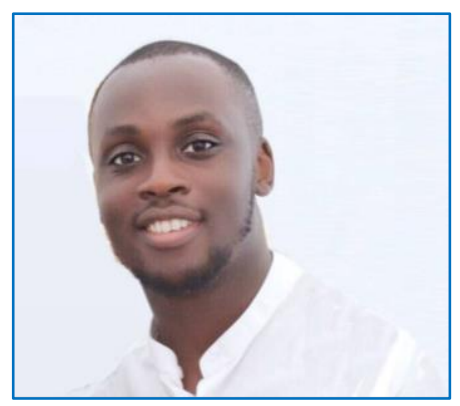

Dr frank OwusuSekyere is a Lecturer in Sport and Exercise Psychology at Kingston University. He has been awarded a PhD, MSc in Sport and Exercise Psychology and a BSc in Sport, Health and Exercise Sciences, all by Brunel University. Frank's research focuses on understanding the nature, development and maintenance of safeguarding cultures in sport. His PhD research captured the impacts of organisations working towards International Safeguards for Children in Sport.

Daichi Ando was awarded a MSc in Sport and Exercise Psychology by Brunel University. Daichi has extensive experience of the sport of rowing. He participated as athlete in various University context. He then went on to coach rowing and contribute to the organisation of rowing events at the University and elite levels 


\section{Introduction}

Coaching plays a critical role in sport through facilitating the development, performance and wellbeing of athletes $[1,2]$. Central to effective coaching is the relationship between the coach and athlete $[3,4]$. A significant body of literature provides empirical evidence of how the quality of the coach-athlete relationship is associated with a range of intrapersonal $[5,6]$, interpersonal [7-9] and group-related $[10,11]$. The effectiveness of this relationship is shaped by a range of intrapersonal, interpersonal and contextual factors [12]. There remains a need to explore the influence of cultural factors in shaping how relationship quality can be maintained and the impact of this on key outcomes [13]. The present study will address this need by exploring relationship maintenance and athlete satisfaction amongst university rowers in the United Kingdom (UK) and Japan.

The coach-athlete relationship has been defined as '...the situation in which coaches' and athletes' emotions, thoughts, and behaviors are mutually and causally inter-connected' [14]. This definition emphasises the bi-directional nature of such relationships and the affective, cognitive, and behavioural interpersonal aspects of coach-athlete relationships.

\subsection{Conceptualising relationship quality}

The most widely cited conceptualisation of the quality of the coach-athlete relationship was proposed by Jowett (2007) [15]. The $3+1 \mathrm{C}$ conceptualisation identifies the key constructs of closeness, commitment, complementarity and co-orientation [15]. Closeness represents feeling emotionally close with one another in the coach-athlete relationship. Commitment refers to coaches' and athletes' shared goals and an intention to work together in the future. Complementarity refers to coaches' and athletes' complementary or co-operative interactions, especially during training. The ' $+1 C^{\prime}$ ' refers to co-orientation which is the degree to which an athlete and coach can accurately infer how his/her coach/athlete is feeling, thinking, and behaving [15].

A range of quantitative studies have demonstrated how relationship quality is associated with key outcomes. These include intrapersonal factors such as exhaustion [5], satisfaction [16] and selfconcept [17]. Relationship quality is also associated with interpersonal factors such as conflict [8], group cohesion [10] and collective efficacy [18].
In the first study to directly investigate how the quality of the coach-athlete relationship can be maintained, Rhind and Jowett (2010) interviewed coaches and athletes from a range of competitive levels and sports [19]. Participants were invited to think about times when they were close, committed, complementary, or co-oriented with their coach/athlete and to talk about the strategies employed to develop and maintain such a relational situation. Based on this interview data, the Coach-Athlete Relationship Maintenance Questionnaire (CARM-Q) was developed to measure the use of 7 relationship maintenance strategies: Conflict management, openness, motivational, preventative, assurance, support and social networks [7]. Conflict Management concerns how a coach and athlete identify, discuss, resolve, and monitor potential areas of conflict within their relationship. Openness relates to how a coach and athlete share relevant information and maintain a good level of communication. The motivational strategy involves a coach and athlete maintaining a reason for continuing the relationship (e.g., setting and achieving goals, making the interactions enjoyable). Preventative strategies are used when a coach and athlete adopt a proactive approach to clarifying and managing expectations. Assurance concerns the extent to which the coach and athlete believe that the other person would be there for them if required and Support relates to the actual provision of support when this was required in the past. Finally, Social Networks involves the maintenance of the broader set of relationships around the coach and athlete (e.g., other players, support staff). These seven relationship strategies form the COMPASS model of relationship maintenance in coach-athlete dyads (acronym of the first letters of the relationship maintenance strategies). Overall, the COMPASS model suggests that the use of these strategies helps to maintain the quality of a coachathlete relationship $[19,20]$. While this model has advanced understanding of maintenance strategies in the coach-athlete relationship, there is a lack of related research in different cultural contexts.

There is a growing body of evidence which demonstrates the importance of considering contextual factors. For example, athletes who participate in individual sports have been found to be closer and more committed to their coach relative to athletes from team sports [21]. Athletes from individual sports also perceived their coach's levels of closeness, commitment and complementarity significantly more highly than athletes from team sports [21]. 
One social factor that has received less attention is the country and culture within which the coach-athlete interact $[12,13]$. One study examined the coach-athlete relationship in seven countries. It found that athletes in Greece and China (typical collectivistic countries) recorded higher levels of closeness and commitment relative to athletes from more individualistic countries [22]. Chelladurai et al., (1988) found that Japanese athletes preferred and perceive more autocratic and supportive leadership styles, while Canadian athletes were found to prefer and perceive a democratic and task-oriented leadership style [23]. Therefore, according to these studies, cultural factors may influence the quality and maintenance of the coach-athlete relationship, but further research is merited to elucidate these impacts in different sports and across different cultures. The present study addresses this gap in the literature through comparing perceptions of athletes in Japan and the UK. Japan is often studied as an example of a collectivistic culture and is typically compared to the United States of America or Canada as examples of a more individualistic culture [23]. The present study focused on athletes in the UK as a different example of a more individualistic culture.

\subsection{Rowing}

The present study focuses on the sport of rowing. There has been a limited amount of related rowing-specific research and this has primarily focused on gender. For example, De Haan and Norman (2019) explored the interpersonal dynamics between female rowers and male coaches [24]. They highlighted evidence of gendered expectations which influenced their roles and perceived capabilities. In another study, De Haan and Knoppers (2020) interviewed elite rowing coaches [25]. They identified that gendered discourses were communicated by coaches which framed female athletes as inferior to the male athletes. There therefore remains scope for further research regarding the coach-athlete relationship in rowing which considers the influence of factors other than gender.

The present study addresses the need for more cross-cultural research regarding the coach-athlete relationship. Specifically, this research poses 4 research questions:

1. How do the coach's use of relationship maintenance strategies differ between rowers in the UK and Japan?

2. How does athlete satisfaction differ between rowers in the UK and Japan?
3. How are the coach's use of relationship maintenance strategies associated with athlete satisfaction in the UK?

4. How are the coach's use of relationship maintenance strategies associated with athlete satisfaction in Japan?

In order to highlight any cultural influence, this study focused on University level rowing to ensure that the competitive level and sport were standardised as far as possible. Such research is important due to the potential for the findings to inform coach education, policy and practice. It is also merited from a research perspective through exploring the extent to which the COMPASS model has validity in different contexts.

\section{Methods}

\subsection{Participants}

A sample of 93 respondents who participated in a university rowing team took part in this study. Of those, 49 (24 male and 25 female) athletes participated in Japan, and 44 (22 male, 22 female) participated in the UK. The participants in Japan were aged 18 to 22 years old $(M=20.31, S D=1.21)$. The average length of their relationship with their principal coach was 1.7 years, and $2.0 \%$ of their coaches were female ( 48 male, 1 female). The average number of years of being a rower was 4.8 years $(S D=2.20)$. The participants in the UK were aged 18 to 26 years old (M $=21.41, \mathrm{SD}=1.95)$, the average length of their relationship with their principal coach was 0.8 years, and $9.1 \%$ of their coaches were female (40 male, 4 female). The average number of years of being a rower was 3.4 years $(S D=2.91)$. All participants were registered as university students, members of the rowing team and had someone who had been coaching them on a weekly basis for at least 6 months.

\subsection{Instrumentation}

All participants completed the surveys in their native language. The 28 item Coach-Athlete Relationship Maintenance Questionnaire (CARM-Q) was utilised to measure the use of the maintenance strategies within the coach-athlete relationship [7]. The cronbach's alpha and an example item is provided for each sub-scale as follows: Conflict Management (5 items, a $=0.68$, e.g., "My coach tries not to lose his/her temper during disagreements"), Openness (4items, a $=0.73$, e.g., "My coach states his/her opinion when we are setting goals"), Motivational (5 items, $a=0.87$, e.g., "My coach shows that $s /$ he is 
motivated to work hard with me"), Preventative (4 items, $a=0.77$, e.g., "My coach tells me what s/he expects from me"), Assurance (3 items, $a=0.73$, e.g., "My coach shows me that I can rely on him/her even when things are not going well"). Support (3 items, a $=0.71$, e.g., "My coach gives me support when I am going through difficult times"), Social Network (4 items, $a=0.78$, e.g., "My coach likes to spend time with our mutual friends"). All CARM-Q items were measured on a likert-type 7-point scale ranging from 1 ("Strongly Disagree") to 7 ("Strongly Agree"). The CARM-Q has demonstrated sound psychometric properties in previous studies within both the UK and Japan $[7,26]$.

The Athlete Satisfaction Questionnaire (ASQ) was utilised to assess four factors: satisfaction with ability utilisation, satisfaction with strategy, satisfaction with personal treatment and satisfaction with training and instruction [27]. The ability utilisation subscale (a $=0.89$ ) contains five items (e.g., 'the degree to which my abilities are (were) used'). The strategy subscale (a $=0.89$ ) contains six items (e.g., 'the coach's choice of plays during competitions'). The personal treatment subscale $(a=0.93)$ contains five items (e.g., 'the recognition I receive (received) from my coach'). Finally, the training and instruction subscale ( $a=0.91)$ contains three items (e.g., 'the training I receive from the coach during the season'). All ASQ items were measured on a likert-type 7-point scale ranging from 1 ("Not at all Satisfied") to 7 ("Extremely Satisfied"). The ASQ has demonstrated sound psychometric properties in a wide range of contexts [27].

\subsection{Procedure}

Following ethical approval from the University Research Ethics Committee, an online version of the participant information sheet, consent form and the questionnaires were created. This explained the purpose of the research, the voluntary nature of participation as well as key ethical considerations related to anonymity, confidentiality and the right to withdraw. All documentation was reviewed by 3 researchers from beyond the project team who spoke both Japanese and English to ensure that the process was clear and easily understood. Data were collected between May-June 2020, which was at the end of the academic year for these student participants.

The head coach of 5 leading University rowing clubs were contacted in both the UK and Japan. All coaches agreed to facilitate the research and hence the participant information sheet, ethics documentation and a link to the online survey were e-mailed to all of the rowers in these clubs. All responses were securely stored on password protected computers.

\subsection{Data analysis}

The data were entered and analysed using version 25 of SPSS by IBM. Firstly, means and standard deviations were calculated for each sub-scale and broken down by the participants from the UK and Japan. Unpaired t-tests were conducted to identify whether there were significant differences between athletes in Japan and the UK in terms of the use of maintenance strategies and athlete satisfaction. Bivariate correlations were utilised to examine whether maintenance strategies were associated with athlete's satisfaction by country.

\section{Results}

\subsection{Preliminary analysis}

The data was screened first. Outliers were identified through visual examination of the box plots and then replaced by rearranging the z-score. Values of skewness and kurtosis were $<| \pm 2|$ across the groups for all 7 CARM-Q items and 4 ASQ items, and Mardia's coefficient value were above 5.0 in both groups, suggesting that the data were normally distributed [28].

RQ1: How do the coach's use of relationship maintenance strategies differ between rowers in the UK and Japan?

Unpaired T-tests were conducted to compare the coach's use of maintenance strategies between the rowers in the UK and Japan (see Table 1). Mean scores for participants in the UK were significantly higher in relation to Conflict Management (t $(91)=5.78, p<$ $.001)$, Openness ( $\mathrm{t}(91)=2.38, p<.05)$, Motivational $(\mathrm{t}(91)=5.26, p<.001)$, Assurance $(\mathrm{t}(84.83)=6.46$, $p<.001)$ and Support strategies ( $\mathrm{t}(91)=-5.98, p<$ $.001)$. This indicates that athletes in the UK perceived that their coaches were more likely to use these strategies. In contrast, mean scores for participants in Japan were significantly higher in Preventative strategies $(\mathrm{t}(91)=2.05, p<.05)$, suggesting that their coaches were more likely to use this strategy. There was no significant difference in the use of the social network strategy.

RQ2: How does athlete satisfaction differ between rowers in the UK and Japan? 
Table 1 Descriptive statistics and unpaired T-test of CARM-Q and ASQ subscales.

\begin{tabular}{lcccc}
\hline & Country & Mean & SD & t-value \\
\hline Conflict Management & JP & 23.18 & 4.92 & $5.78^{* * *}$ \\
& UK & 28.70 & 4.22 & \\
Openness & JP & 17.14 & 4.87 & $2.38^{*}$ \\
Motivational & UK & 19.48 & 4.54 & \\
& JP & 24.41 & 6.07 & $-5.26 * * *$ \\
Preventative & UK & 30.23 & 4.37 & \\
& JP & 15.73 & 5.64 & $2.05^{*}$ \\
Assurance & UK & 13.52 & 4.67 & \\
Support & JP & 10.31 & 4.40 & $-6.46 * * *$ \\
& UK & 15.30 & 2.98 & \\
Social Networks & JP & 9.76 & 4.26 & $-5.93 * * *$ \\
& UK & 14.48 & 3.22 & \\
Ability Utilization & JP & 14.65 & 5.54 & -1.01 \\
& UK & 15.91 & 6.42 & \\
Strategy & JP & 22.33 & 7.06 & $-3.39 * * *$ \\
& UK & 26.93 & 5.89 & \\
Personal Treatment & JP & 24.88 & 7.85 & $-3.44 * * *$ \\
& UK & 30.16 & 6.86 & \\
Training and & JP & 21.88 & 8.48 & $-2.61 *$ \\
Instruction & UK & 26.05 & 6.71 & \\
\hline Note: $* \mathrm{p}<.05$, & JP & 13.00 & 4.47 & $-3.21 * *$ \\
$* * p<.01$, & UK & 15.70 & 3.54 & \\
$* * * p<.001$ & & & & \\
& & & & \\
& & & &
\end{tabular}

Table 2 Bivariate Correlation of CARM-Q and ASQ subscales for Japan $(n=49)$

\begin{tabular}{lcccc}
\hline & Ability Utilization & Strategy & $\begin{array}{c}\text { Performance } \\
\text { Treatment }\end{array}$ & $\begin{array}{c}\text { Training and } \\
\text { Instruction }\end{array}$ \\
\hline Conflict & $.399^{* *}$ & .247 & $.383^{* *}$ & $.358^{*}$ \\
Management & & & & $.667^{* *}$ \\
Openness & $.653^{* *}$ & $.702^{* *}$ & $.635^{* *}$ \\
Motivational & $.732^{* *}$ & $.780^{* *}$ & $.705^{* *}$ & $.668^{* *}$ \\
Preventative & $.572^{* *}$ & $.682^{* *}$ & $.595^{* *}$ & $.552^{* *}$ \\
Assurance & $.517^{* *}$ & $.597^{* *}$ & $.500^{* *}$ & $.485^{* *}$ \\
Support & $.630^{* *}$ & $.607^{* *}$ & $.612^{* *}$ & $.544^{* *}$ \\
Social Networks & $.569 * *$ & $.600^{* *}$ & $.519 * *$ & $.521^{* *}$ \\
\hline
\end{tabular}

$* \mathrm{p}<.05, * * \mathrm{p}<.01$

Table 3 Bivariate Correlation of CARM-Q and ASQ subscales for the UK $(n=44)$

\begin{tabular}{lcccc}
\hline & Ability Utilization & Strategy & $\begin{array}{c}\text { Performance } \\
\text { Treatment }\end{array}$ & $\begin{array}{c}\text { Training and } \\
\text { Instruction }\end{array}$ \\
\hline Conflict & .162 & 0.34 & .190 & .024 \\
Management & $.522^{* *}$ & $.535^{* *}$ & $.488^{* *}$ & $.578^{* *}$ \\
Openness & $.352^{*}$ & $.299^{*}$ & .279 & $.311^{*}$ \\
Motivational & $.252^{* *}$ & $.385^{* *}$ & .295 & $.539^{* *}$ \\
Preventative & $.345^{*}$ & $.371^{*}$ & $.337^{*}$ & $.421^{* *}$ \\
Assurance & $.417^{* *}$ & $.372^{*}$ & $.440^{* *}$ & $.343^{*}$ \\
Support & $.347^{*}$ & $.390^{* *}$ & $.388^{* *}$ & $.403^{* *}$ \\
Social Networks & & & &
\end{tabular}

$* \mathrm{p}<.05, * * \mathrm{p}<.01$ 
Unpaired T-tests were conducted to compare levels of athlete satisfaction between the rowers in the UK and Japan (see Table 1). Mean scores for participants in the UK were significantly higher in relation to Ability Utilization ( $\mathrm{t}(91)=3.39, \mathrm{p}<.01)$, Strategy $(\mathrm{t}(91)=3.43, \mathrm{p}<.001)$, Performance Treatment $(\mathrm{t}(91)=2.61, \mathrm{p}<.05)$, and Training and Instruction $(\mathrm{t}(91)=3.21, \mathrm{p}<.01)$. This indicates that rowers in the UK were more satisfied across all of these areas relative to rowers in Japan.

RQ3: How are the coach's use of relationship maintenance strategies associated with athlete satisfaction in the UK?

Bivariate correlations were conducted between the coach's use of each relationship maintenance strategy and the different aspects of satisfaction amongst the athletes in the UK. All aspects of athlete satisfaction were significantly correlated with the use of multiple maintenance strategies. Overall, the strongest relationship was with the coach's use of the openness strategy for all aspects of satisfaction. However, no significant associations were found with the coach's use of conflict management.

Focusing specifically on each factor, Ability Utilisation was also strongly associated with the coach's use of support. Satisfaction with the coach's strategy was correlated with the coach's use of preventative and social network strategies. Personal Treatment was associated with support and social networks. Finally, the satisfaction with Training and Instruction was correlated with preventative, assurance and social networks strategies.

RQ4: How are the coach's use of relationship maintenance strategies associated with athlete satisfaction in Japan?

Bivariate correlations were conducted between the coach's use of each relationship maintenance strategy and the different aspects of satisfaction amongst the athletes in Japan. All aspects of athlete satisfaction were significantly correlated with the coach's use of multiple maintenance strategies. Overall, the strongest relationships were between the use of motivational and openness strategies for all aspects of satisfaction. The coach's use of support was also strongly correlated with the athlete's satisfaction regarding Ability Utilisation and Personal treatement. The coach's use of the Preventative strategy was also found to be strongly associated with the athlete's satisfaction with the coach's strategy as well as training and instruction.
In comparing the two sets of correlations, it is noteworthy that the relationships were stronger between maintenance strategies and satisfaction amongst rowers in Japan relative to those in the UK. Furthermore, the coach's use of Conlfict management was found to be significantly associated with Ability Utilisation, Personal Treatment, and Training and Instruction in Japan compared to no significant correlations in the UK sample.

\section{Discussion and Conclusion}

The present study explored how the coach's perceived use of relationships maintenance strategies and athlete satisfaction differed between rowers in the UK and Japan. The associations between maintenance strategies and satisfaction within the two locations was also analysed. Overall, this research serves to illustrate that there are indeed cross-cultural variations and hence cultural factors merit consideration. This supports recent theoretical perspectives which have advocated for the importance of contextual influences when analysing coach-athlete [12, 13]. Furthermore, strong associations were found between maintenance strategies and satisfaction in both the UK and Japan. This finding provides empirical support for the COMPASS model. Each of the four research questions are now discussed in turn.

When comparing the use of the strategies, coaches in the UK were perceived to use conflict management, openness, motivational, assurance and support significantly more often. Coaches in Japan were significantly more likely to be perceived to use the preventative strategy, which may be a proactive approach to avoid conflict $[8,9]$. These results are consistent with the previous research which found that Japanese participants tend to avoid conflict in interpersonal relationships more than American participants [29]. In terms of athlete satisfaction, rowers in the UK reported significantly higher scores for all of the different elements, specifically Ability Utilization, Strategy, Performance treatment, as well as Training and Instruction. This finding is consistent with previous research which has found that the level of satisfaction experienced by athletes regarding the leadership behaviours of their coach in Japan is less than in western countries [23]. This highlights the need for further research to explore the feasibility of encouraging the use of more maintenance strategies by coaches as well as the subsequent impact on satisfaction. 
Considering the associations between maintenance strategies and satisfaction, the coach's use of openness appeared to be a particularly important factor across all athletes. This lends support to the argument that open lines of communication are the fuel which powers effective coach-athlete relationships [1]. In the UK, the coach's use of support and social network strategies were also found to be strongly associated with satisfaction. In Japan, the coach's use of motivational and preventative strategies was found to be strongly associated with satisfaction. In comparing the two sets of correlations, it is noteworthy that the relationships were stronger between maintenance strategies and satisfaction amongst rowers in Japan relative to those in the UK. Furthermore, the coach's use of conflict management was found to be significantly associated with Ability Utilisation, Personal Treatment, and Training and Instruction in Japan compared to no significant correlations in the UK sample.

These cultural differences may be explained from a cross-cultural perspective. The findings could be a result of the differences between the more individualistic culture (UK) and the more collectivistic culture (Japan). The related concepts of the interdependent self and the independent self is also relevant [30]. People in collectivistic cultures tend to emphasize the importance of cohesiveness among individuals. As an example of typical Japanese characteristics, there is a Japanese word for self, jibun. This refers to "one's share of the shared life space" [31]. According to Hamaguchi (1985), selfness is only confirmed through interpersonal relationships [31]. The use of motivational and preventative strategies were strongly associated with satisfaction. This may reflect the cultural importance of promoting a cohesive relationship and avoiding interpersonal conflict in Japan [32].

\section{Limitations and Future Research}

There are some limitations of the present study which need to be acknowledged. Firstly, one cannot make inferences about causality based on this cross-sectional data. More longitudinal research and the evaluation of interventions is required to investigate the directions of these relationships. For example, an educational intervention to promote the use of maintenance strategies amongst a group of coaches could be provided with measures taken of relationship quality, the use of the strategies and athlete satisfaction before and after the intervention. Follow-up measures could also be taken to assess the retention of any impacts. This could be compared against a matched control group of coaches. Secondly, coaches were not included in the present study and hence there is a need to collect data at the dyadic level. The data collected in the present study focused on the perceived use of maintenance strategies and hence other methods could be used to analyse the actual use of maintenance strategies, such as through systematic observations. Finally, only satisfaction was measured as one important outcome and hence there is considerable scope to explore how the use of maintenance strategies are associated with other important outcomes such as performance, well-being and group cohesion.

Overall, this research highlights the importance of considering cultural factors and the influence of context. Significant associations between the coaches perceived use of maintenance strategies and athlete satisfaction has been demonstrated in both the UK and Japan. This empirical evidence provides further support for the COMPASS model proposed by Rhind and Jowett (2010) [19]. It serves to highlight the need for more cross-cultural research which can inform policies, education and practice to promote the maintenance of effective relationships between coaches and athletes. This can in turn provide the foundation for athletic success and personal development.

\section{References}

[1] L. Davis, S. Jowett, S. Tafvelin, Communication strategies: The fuel for quality coach-athlete relationships. Frontiers in Psychology, 10(2019) 21-56. [DOI] [PubMed]

[2] W. Gilbert, Coaching Better Every Season: A Year-Round Process for Athlete Development and Program Success, Human Kinetics, (2017) Champaign, IL

[3] C. Cronin, K. Armour, (Eds.) Care in Sport Coaching: Pedagogical Cases (1st ed.), (2018) Routledge, UK. [DOI]

[4] S. Jowett, Coaching effectiveness: the coachathlete relationship at its heart. Current opinion in psychology, 16(2017) 154-158. [DOI] [PubMed]

[5] L. Davis, R. Appleby, P. Davis, M. Wetherell, H. Gustafsson, The role of coach athlete relationship quality in team sport athletes' psychophysiological exhaustion: implications for physical and cognitive performance, Journal of Sports Sciences, 36(2018) 1985-1992. [DOI] [PubMed] 
[6] S. Isoard-Gautheur, D. Trouilloud, H. Gustafsson, E. Guillet-Descas, Associations between the perceived quality of the coachAthlete relationship and athlete burnout: an examination of the mediating role of achievement goals, Psychology of Sport and Exercise, 22(2016) 210-217. [DOI]

[7] D.J.A. Rhind, S. Jowett, Development of the Coach-Athlete Relationship Maintenance Questionnaire (CARM-Q), International Journal of Sports Science \& Coaching, 7(1) (2012) 121-137. [DOI]

[8] S. Wachsmuth, S. Jowett, C. Harwood, On understanding the nature of interpersonal conflict between coaches and athletes, Journal of Sports Sciences 36(17) (2018) 1955-1962. [DOI] [PubMed]

[9] S. Wachsmuth, S. Jowett, C.G. Harwood, Conflict among athletes and their coaches: what is the theory and research so far?, International Review of Sport and Exercise Psychology, 10(1) (2017) 84-107. [DOI]

[10] S. Jowett, V. Chaundy, (2004). An Investigation Into the Impact of Coach Leadership and Coach-Athlete Relationship on Group Cohesion. Group Dynamics: Theory, Research, and Practice, 8(4), 302-311. [DOI]

[11] S. Jowett, V. Shanmugam, Relational coaching in sport: its psychological underpinnings and practical effectiveness, in Routledge International Handbook of Sport Psychology, eds R. Schinke, K. R. McGannon, and B. Smith (Abingdon: Routledge), (2016) 471-484.

[12] D.J.A. Rhind, F. Owusu-Sekyere, International Safeguards for Children in Sport: Developing and embedding a safeguarding culture, Routledge, (2020) London.

[13] F. Owusu-Sekyere, D.J.A Rhind, L. Hills, (2021). Safeguarding Culture: Towards a new approach to preventing child maltreatment in sport, Sport Management Review, 1-23. [DOI]

[14] S. Jowett, N. Ntoumanis, The Coach - Athlete Relationship Questionnaire (CART - Q): Development and initial validation. Scandinavian Journal of Medicine and Science in Sports, 14(2004) 245-257. [DOI]

[15] S. Jowett, Interdependence analysis and the $3+1 \mathrm{Cs}$ in the coach-athlete relationship, In Jowett S. \& Lavallee D. (Eds.), Social psychology in sport, Human Kinetics, (2007) 15-27.
[16] S. Jowett, J. Nezlek, Relationship interdependence and satisfaction with important outcomes in coach-athlete dyads, Journal of Social and Personal Relationships, 29(3) (2012) 287-301. [DOI]

[17] S. Jowett, Moderators and mediators of the association between the coach-athlete relationship and physical self-concept, International Journal of Coaching Science, 2(2008) 43-62.

[18] R. Hampsom, S. Jowett, Effects of coach leadership and coach-athlete relationship on collective efficacy, Scandinavian Journal of Medicine and Science in Sport, 24(2014) 454460. [DOI] [PubMed]

[19] D.J.A. Rhind, S. Jowett, Relationship Maintenance Strategies in the Coach-Athlete Relationship: The Development of the COMPASS Model, Journal of Applied Sport Psychology, 22(1) (2010) 106-121. [DOI]

[20] D.J.A. Rhind, S. Jowett, Linking maintenance strategies to the quality of coach-athlete relationships, International Journal of Sport Psychology, 42(2011) 1-14.

[21] D.J.A. Rhind, S. Jowett, S.X. Yang, A comparison of athletes' perceptions of the coach-athlete relationship in team and individual sports, Journal of Sport Behavior, 35(4) (2012) 433-452.

[22] S. Yang, S. Jowett, Psychometric properties of the Coach-Athlete Relationship Questionnaire (CART-Q) in seven countries, Psychology of Sport and Exercise, 13(2012) 36-43. [DOI]

[23] P. Chelladurai, H. Imamura, Y. Yamaguchi, Y. Oinuma, T. Miyauchi, (1988). Sport Leadership in a Cross-National Setting: The Case of Japanese and Canadian University Athletes, Journal of Sport and Exercise Psychology, 10(4), 374-389. [DOI]

[24] D. De Haan, L. Norman, Mind the gap: The presence of capital and power in the female athlete-male coach relationship within elite rowing, Sports Coaching Review, 9(1) (2020) 95-118. [DOI]

[25] D. De Haan, A. Knoppers, Gendered discourses in coaching high-performance sport, International Review for the Sociology of Sport, 55(6) (2020) 631-646. [DOI]

[26] C. Kuribayashi, H. Sato, Pilot evaluation of psychometric property of the Coach-Athlete Relationship Maintenance Questionnaire in Japanese junior tennis players. Japanese 
Journal of Sport Psychology, 4(2) (2015) 93102. [DOI]

[27] H.A. Riemer, P. Chellandurai, Development of the Athlete Satisfaction Questionnaire (ASQ). Journal of Sport \& Exercise Psychology, 20(2) (1998) 127-156. [DOI]

[28] AP. Field, Discovering statistics using SPSS (2nd ed.), (2005) Sage Publications, London.

[29] K.-I. Ohbuchi, Y. Takahashi, Cultural styles of conflict management in Japanese and Americans: Passivity, covertness, and effectiveness of strategies, Journal of Applied Social Psychology, 24(15) (1994) 1345-1366. [DOI]

[30] T. Sawaumi, S. Yamaguchi, J. Park, A.R. Robinson, Japanese Control Strategies Regulated by Urgency and Interpersonal Harmony: Evidence Based on Extended Conceptual Framework, Journal of CrossCultural Psychology, 46(2) (2015) 252-268. [DOI]

[31] E. Hamaguchi, A contextual model of the Japanese: Toward a methodological innovation in Japan studies, Journal of Japanese Studies, 11(2) (1985) 289-321. [DOI]

[32] E.J. Kim, A. Yamaguchi, M.S. Kim, \& A. Miyahara, Effects of taking conflict personally on conflict management styles across cultures. Personality and Individual Differences, 72(2015) 143-149. [DOI]

\section{Acknowledgement}

The author received no financial support for the research, authorship, and/or publication of this article.

\section{Funding}

No funding was received to carry out this study

\section{Authors Contribution}

Dr. Rhind - Conceptualization, study design and Draft preparation; Dr. Owusu-Sekyere - Draft preparation. Mr. Ando - Study design, data collection and analysis. All the authors read and approved the final version of the draft.

\section{Ethics Approval}

Approval was sought from the University Research Ethics Committee

\section{Informed Consent}

Written consent was obtained from the participants

\section{Conflict of interest}

The Authors have no conflicts of interest to declare that they are relevant to the content of this article.

\section{Does this article screened for similarity? Yes}

\section{About The License}

(c) The author(s) 2021. The text of this article is open access and licensed under a Creative Commons Attribution 4.0 International License 